\title{
Analysis of Housing Risk Factors for the Welfare of Lean and Heavy Pigs in a Sample of European Fattening Farms
}

\author{
Paolo Ferrari $^{1, *}$, Alessandro Ulrici ${ }^{2}$ (D) and Matteo Barbari ${ }^{1}$ (D) \\ 1 Department of Agriculture, Food, Environment and Forestry (DAGRI), University of Florence, \\ P.le delle Cascine, 18, 50144 Firenze, Italy; matteo.barbari@unifi.it \\ 2 Department of Life Sciences, University of Modena and Reggio Emilia, Via Amendola, 2, \\ 42122 Reggio Emilia, Italy; alessandro.ulrici@unimore.it \\ * Correspondence: paolo.ferrari@unifi.it; Tel.: +39-347-965-3445
}

Citation: Ferrari, P.; Ulrici, A.;

Barbari, M. Analysis of Housing Risk

Factors for the Welfare of Lean and

Heavy Pigs in a Sample of European Fattening Farms. Animals 2021, 11,

3221. https://doi.org/10.3390/

ani11113221

Academic Editor: Emma Fàbrega i Romans

Received: 9 October 2021

Accepted: 10 November 2021

Published: 11 November 2021

Publisher's Note: MDPI stays neutral with regard to jurisdictional claims in published maps and institutional affiliations.

Copyright: (c) 2021 by the authors. Licensee MDPI, Basel, Switzerland. This article is an open access article distributed under the terms and conditions of the Creative Commons Attribution (CC BY) license (https:// creativecommons.org/licenses/by/ $4.0 /)$.
Simple Summary: Animal welfare is a major challenge that most European pig producers have been facing in recent decades to comply with EU legislation and to meet the increasing societal and market demand for pork produced in a sustainable way. Pig welfare is ruled in terms of minimum requirements for housing and management, but stakeholders have considered that both farm-level and animal-based indicators are fundamental to monitor animal welfare. Some of the welfare issues still affecting fattening pigs are the lack of space, bedding and manipulable material, and the continued practice of routine tail docking of pigs. Tail docking is applied routinely across most European countries to reduce the occurrence of severe tail biting lesions, despite its ban in the EU. An observational study on 51 pig farms in seven EU countries, aimed at investigating housing risk factors for the welfare of finishing pigs, showed that body weight and presence of bedded solid floored resting area (BED) identify three clusters of farms. The outcomes of this study confirmed that BED and larger availability of space per pig, above the minimum requirement of EU legislation, can limit the occurrence of lesions in pigs with undocked tails.

Abstract: Pig welfare is affected by housing conditions, the minimum requirements of which are set up by EU legislation. Animal and non-animal-based measures are useful indicators to investigate housing risk factors for pig welfare. An observational study on 51 pig farms in seven EU countries, aimed at investigating housing risk factors for the welfare of finishing pigs, showed body weight and presence of bedded solid floored resting area (BED) identifying three clusters of farms. Farms with BED were featured by no or limited tail docking, larger availability of manipulable materials and lower number of pigs per farm and per annual work unit. In these farms, less skin and ear lesions were found, compared with lean pigs of farms without BED, which were characterized by lower pig space allowance, mortality rate and medication cost. In farms without BED, heavy pigs were featured by more space per pig, more pigs per drinker and higher mortality rate and medication cost per pig, compared to lean pigs. No statistical difference in tail lesions was found between the three farm clusters, although tail docking was performed in all farms without BED and not performed on most farms with BED.

Keywords: housing system; pig welfare; fattening pig; body lesion scores; bedding material; enriched environment; roughage; tail docking

\section{Introduction}

Animal welfare is a major challenge that most European pig producers have been facing in recent decades to comply with EU legislation and to meet the increasing societal and market demand for pork produced in a sustainable way. Although pig welfare has been governed by EU legislation since 1991 [1], some major welfare issues still remain, such as the lack of space allowance, enrichment materials and bedding, and the practice of 
tail docking if carried out routinely [2]. Housing conditions are deemed by stakeholders as particularly important to safeguard animal welfare, as well as the use of animal-based and farm-level indicators to monitor the progress of animal welfare [3].

Animal-based measures were developed to directly assess the effective welfare state of the pig by measuring, for example, its behaviour, fearfulness, health, or physical condition [4]. Nevertheless, the European legislation for the protection of pigs is based on housing and management risks which can be assessed by using resource-based measures (i.e., non-animal-based measures) rather than animal-based measures [5]. Resource-based measures are indirect measures of animal welfare because measuring the ability of the farming system (housing and management) to provide pigs with conditions to which the pigs can adapt without endangering their welfare; therefore, monitoring resource-based measures can be useful to identify risk factors that lead or may lead to actual welfare problems in pigs, which can be measured by animal-based measures. Therefore, monitoring both animal-based and non-animal-based measures is a promising approach to advice pig farmers to control and improve the welfare conditions of pigs. "Age of the animals, type of floor, feeding system, stocking density and environmental temperature can be useful to predict the appearance of a given welfare measure of 'good housing' on a farm" [6,7].

European legislation sets to $1 \mathrm{~m}^{2} /$ pig the minimum floor space allowance for pigs over $110 \mathrm{~kg}$ of body weight without any further indication for heavier pigs, such as the Italian heavy pigs slaughtered at the minimum age of 9 months and at the live weight of $160 \mathrm{~kg} \pm 10 \%$, according to the Parma ham Protected Designation of Origin (PDO) scheme [8]. The allometric equation $\mathrm{A}=\mathrm{k} \times \mathrm{BW}^{0.67}$ was used with $\mathrm{k}=0.03$ to calculate the minimum legal space allowance for growing and fattening pigs [9] although EFSA recommended $\mathrm{k}=0.036$ for pigs up to $110 \mathrm{~kg}$ of live weight and $\mathrm{k}=0.047$ above $110 \mathrm{~kg}$, to allow all pigs to rest simultaneously in lateral lying posture [10]. One study showed that less stocking densities and reduced pen size can lead to more pigs laying at the same time, less pig lesions, less pen dirtiness and higher average daily growth [11].

The influence of group size on pig welfare is controversial; no significant effect was proved on fattening pigs according to some authors [12-15], whereas an increase in group size would result into unfavourable effects on welfare and performance, according to other studies [11].

Pig dunging behaviour is affected by space allowance because different functional areas are used by pigs for resting and for dunging, unless the pigs are heat stressed or sick or the stocking density is too high [16]. Pig and litter cleanliness in straw bedded pens was also found as negatively affected by hot climate [17]. One study showed that pen soiling increases with increasing age in pigs kept on solid floor, as floor soiling and wallowing behaviour was more prevalent in the late growth period [18]. More pig soiling was observed in pigs that were liquid fed, compared to pigs fed with wet and dry feed [7]. Italian heavy pigs are traditionally liquid fed during the entire growing-fattening phase, which is approximately 6 month long [19].

Free access to water of good quality is mandatory in fattening pigs [20] and needed even if liquid or wet feed is provided [16]. To this end, a maximum number of fattening pigs per functioning drinker is recommended, depending on the type of drinker [21]: 12 pigs per nipple or 15 pigs per water bowl.

Slatted floors systems are widely used for pig housing throughout the EU [16]. Fully slatted or partially slatted floors are generally used to house heavy pigs in Italy [19]. They promote pig cleanliness and hygiene by allowing the quick and effective removal of faeces and urine from the pen, although fully slatted floors were found to limit the use of straw as bedding or manipulable material to allow pigs to perform explorative behaviour [16].

Manipulable materials are needed to enrich the pen environment for pigs intensively kept in order to meet their exploration behavioural needs and avoid tail biting and skin lesions in growing and fattening pigs kept at high stocking densities [22]. One study found decreased exploration of enrichment material with increasing live weight [15], 
so special attention should be given to providing effective enrichment as the pigs' live weight increases.

Enrichment materials are categorized as [23]: (a) optimal materials which can be used alone because they are "edible, chewable, investigable, manipulable, of sustainable interest, accessible for oral manipulation, given in sufficient quantity, clean and hygienic"; (b) suboptimal materials, possessing most of the previous characteristics but not all of them so that their use should be combined with other materials; (c) "materials of marginal interest providing distraction for pigs which should not be considered as fulfilling their essential needs".

Straw is considered as one of the best enrichment materials [16]; it has been demonstrated that its distribution in racks in fully slatted housing systems is possible and does not compromise the effectiveness of the manure removal system [24].

Pig welfare is also affected by stockperson's action [16], so the ratio of number of pigs to number of stockpersons was acknowledged as a predictor variable for severe tail lesions in heavy pigs [25].

Most pigs in Europe are tail-docked despite the fact that the practice of routine taildocking was banned in 1994 [1]. Tail docking aims at reducing the frequency of tail biting and the related tail lesions, but it is painful for pigs and can lead to neuroma formation [26].

However, the occurrence of tail biting depends on a wide range of factors such as the lack of environmental enrichment, stocking density, presence of slatted floors, microclimate discomfort, high levels of dust and noxious gases (i.e., ammonia), competition for resources, social instability and genetic, dietary and health factors $[14,26]$. Additional risk factors to predict farms having severe tail lesions were identified in: pig age, live weight at slaughter, space allowance for $100 \mathrm{~kg}$ of live weight, number and type of drinkers, pen size and number of pigs in the farm $[22,25]$.

Tail biting can occur in all production systems, including free-range and organic [27-29]. Particular attention should be paid by farmers keeping pigs with intact tails through frequent observation and timely intervention in case of tail-biting outbreaks, which can spread rapidly and become difficult to stop [30].

The prevalence of physical conditions in pigs varies between herds [27,31]. Tail, skin, and ear lesions are used widely as animal-based measures to directly assess animal welfare of growing and fattening pigs $[4,5]$.

The farm average pig's mortality rate is a common measure of health and welfare for pig herds. Mortality is defined as "the uncontrolled death of animals (as distinct from culling/euthanasia). Any animal which is found dead on the floor in the house, or out on the field is considered a mortality" [4]. Pigs may be culled (i.e., emergency killing) if they are injured or sick to avoid exposing them to severe pain or suffering, or if no other practical way is available to relieve the pain [32]. One study shows that emergency killing is more frequently implemented on piglets rather than on older pigs, such as growing and fattening pigs [33].

\section{Materials and Methods}

An observational study was carried out across seven EU countries by using the Condensed protocol from the Era-Net SusAn project "Sustainable pig production systems" (SusPigSys) [34]. Animal welfare data, together with a number of economic/production data considered as relevant for animal welfare assessment, were included in this study for growing-fattening units involved in the SusPigSys project. For this purpose, 31 non-animal and animal-based measures were considered as animal welfare indicators for both heavy and lean pig farms in the growing and finishing phase, across fattening units of 51 pig farms in seven EU countries (i.e., IT, DE, AT, NL, PL, FI, UK). Type and description of variables are given in Tables 1 and 2. Farm units with uncomplete data were excluded from this study, as well as cases of observed pig groups with uncomplete or inconsistent animal welfare data for one or more variables. Complete data from 51 fattening pig units were processed statistically, including: (1) economic/production data of visited 
farms, considered as potentially relevant for pig welfare (i.e., affecting or affected by pig welfare); (2) animal welfare measures of up to 15 pig groups observed during farm visit, except for three Polish farms in which 16, 17 and 18 pig groups have been observed for more representativity.

Pig group sampling and pig number sizing in each group for collection of animal welfare data were based on the "Real Welfare" scheme strategy [35].

Pig groups were chosen randomly and in proportion of their stage of production: one third of the pig groups at the early fattening period but grouped at least two weeks before farm visit, one third in the middle of the fattening period and one third at the end of the fattening period. For pig groups with 100 or less pigs per group, up to 15 pig groups were assessed by observing up to 50 pigs per group. For groups larger than 100 pigs, at least $50 \%$ of pigs per group were observed up to a total of 750 pigs for all groups. Animal-based information was collected by observing the animals in their own environment from a distance of $50 \mathrm{~cm}$. A total of 709 pig groups from the 51 pig farms were assessed.

Prior to the start of farm visits, training material including definitions was created and assessors were trained at a joint training occasion in order to achieve a consistent scoring, which was tested as inter-assessor agreement (IOR) on-farm for all measures that required scoring, using joint assessments and photo material. IOR was calculated as exact agreement between two observers and expressed as weighted Kappa, PABAK and percentage agreement.

Farmers were recruited on a voluntary basis; before and at the beginning of each farm visit, the farmer (i.e., person(s) to be interviewed) was informed in speech and writing about the project, including information about anonymity, why the research was being conducted, how his or her data were being used and if there were any risks associated, and were asked to return a signed informed consent before the start of data collection. Farmers were asked, before pig observation, about the number of pig houses, pig groups per pig house, pigs per group and related ages, and if no, or some, or all pigs were tail docked.

Table 1. Continuous, ordinal, and dichotomous variables for 709 observed pig groups.

\begin{tabular}{|c|c|c|c|c|}
\hline N. & Variable Description & Acronym & Type $1^{1}$ & Type $2^{2}$ \\
\hline 1 & Total area indoor of observed pens & TAI & nABM & $\mathrm{C}$ \\
\hline 2 & Number of pigs per observed pen & NP & $\mathrm{nABM}$ & $\mathrm{C}$ \\
\hline 3 & Average pig live weight in observed pens & AW & ABM & $\mathrm{C}$ \\
\hline 4 & Mean space allowance $\left(\mathrm{m}^{2}\right)$ per pig in observed pens & $\mathrm{SP}$ & $\mathrm{nABM}$ & $\mathrm{C}$ \\
\hline 5 & Mean space allowance per $100 \mathrm{~kg}$ of pig liveweight in observed pens & SK & ABM & $\mathrm{C}$ \\
\hline 6 & Mean number of pigs per drinker in observed pens & PD & $\mathrm{nABM}$ & $\mathrm{C}$ \\
\hline 7 & $\%$ of active pigs manipulating enrichment in observed pens & EMB & ABM & $\mathrm{C}$ \\
\hline 8 & $\%$ of active pigs in reach of enrichment material in observed pens & PAE & ABM & $\mathrm{C}$ \\
\hline 9 & Laying area dirtiness: $1=$ clean; $2=$ medium; $3=$ dirty & DL & $\mathrm{nABM}$ & $\mathrm{O}$ \\
\hline 10 & Slatted Floor: $0=$ no; $1=$ partial; $2=$ totally slatted & SF & $\mathrm{nABM}$ & $\mathrm{O}$ \\
\hline 11 & Liquid feeding system: $1=$ dry; $2=$ wet or mixed dry $/$ liquid $; 3=$ liquid & LFS & $\mathrm{nABM}$ & $\mathrm{O}$ \\
\hline 12 & $\begin{aligned} \text { Bedding in lying area: } 0=\text { no bedding, } 1=\text { not all pigs can lie on bedded area, } 2=\text { enough in } \\
\text { laying area; } 3=\text { all pen floor bedded }\end{aligned}$ & $\mathrm{BP}$ & nABM & $\mathrm{O}$ \\
\hline 13 & Presence of roughage: $0=$ no roughage; $1=$ pellet; $2=$ straw; $3=$ hay $/$ silage & $\mathrm{RP}$ & nABM & $\mathrm{O}$ \\
\hline 14 & $\begin{array}{l}\text { Presence of enrichment: } 0=\text { no enrichment; } 1 \text { = other enrichment of marginal interest; } \\
2 \text { = suboptimal or optimal/proper enrichment combined with other enrichment; } \\
3=\text { proper/optimal enrichment }\end{array}$ & $\mathrm{EP}$ & nABM & $\mathrm{O}$ \\
\hline 15 & Presence of tail docked pigs: $0=$ no tail docked; $1=$ some tail docked; $2=$ all tail docked & TD & nABM & $\mathrm{O}$ \\
\hline 16 & $\begin{array}{l}\text { Short tail: } 0=\text { no pigs with tail shortened by less than } 50 \% \text { of the original length; } 1=\leq 10 \% \text { pigs } \\
\text { with tails shortened by less than } 50 \% ; 2=>10 \% \text { pigs with tails shortened by less than } 50 \%\end{array}$ & SHT & $\mathrm{ABM}$ & $\mathrm{O}$ \\
\hline 17 & $\begin{array}{l}\text { Tail stump: } 0=\text { no pigs with tail shortened by more than } 50 \% \text { of the original length; } 1=\leq 10 \% \\
\text { pigs with tails shortened by more than } 50 \% ; 2=>10 \% \text { pigs with tails shortened by more than } 50 \%\end{array}$ & STT & $\mathrm{ABM}$ & $\mathrm{O}$ \\
\hline 18 & $\begin{array}{c}\text { Tail lesions: } 0=\text { no; } 1=\leq 10 \% \text { pigs have mild damage, but no pig has severe damage; } 2=>10 \% \\
\text { pigs have mild damage, and/or } 1 \text { has severe damage }\end{array}$ & $\mathrm{T}$ & $\mathrm{ABM}$ & $\mathrm{O}$ \\
\hline 19 & $\begin{array}{c}\text { Ear lesions: } 0=\text { no; } 1=\leq 10 \% \text { pigs have mild damage, but no pig has severe damage; } 2=>10 \% \\
\text { pigs have mild damage, and/or } 1 \text { has severe damage }\end{array}$ & $\mathrm{E}$ & $\mathrm{ABM}$ & $\mathrm{O}$ \\
\hline 20 & $\begin{array}{l}\text { Body lesions: } 0=\text { no skin lesions; } 1=\leq 20 \% \text { pigs have mild skin lesions, but no pig has severe } \\
\text { damage; } 2=>20 \% \text { pigs have mild skin lesions, and /or } 1 \text { has severe damage }\end{array}$ & B & $\mathrm{ABM}$ & $\mathrm{O}$ \\
\hline 21 & Feed restriction $0=$ no, $1=$ yes & FR & $\mathrm{nABM}$ & $\mathrm{D}$ \\
\hline 22 & Outdoor access $0=$ no, $1=$ yes & OA & nABM & $\mathrm{D}$ \\
\hline 23 & Organic farm $0=$ no, $1=$ yes & OR & nABM & $\mathrm{D}$ \\
\hline
\end{tabular}

${ }^{1}$ Animal Based Measure (ABM) or non-Animal Based Measure (nABM). ${ }^{2}$ continuous $(\mathrm{C})$ or ordinal $(\mathrm{O})$ or dichotomous (D) variables. 
Table 2. Continuous, ordinal and dichotomous variables for 51 fattening pig units.

\begin{tabular}{|c|c|c|c|c|}
\hline N. & Variable Description & Acronym & Type $1^{1}$ & Type $2^{2}$ \\
\hline 1 & Farm mean total area indoor of observed pens & TAI & nABM & $\mathrm{C}$ \\
\hline 2 & Farm mean number of pigs in observed pens & NP & $\mathrm{nABM}$ & $\mathrm{C}$ \\
\hline 3 & Average pig live weight in observed pens & $\mathrm{AW}$ & ABM & $\mathrm{C}$ \\
\hline 4 & Farm mean space allowance $\left(\mathrm{m}^{2}\right)$ per pig in observed pens & $\mathrm{SP}$ & $\mathrm{nABM}$ & $\mathrm{C}$ \\
\hline 5 & Farm mean space allowance per $100 \mathrm{~kg}$ of pig live weight in observed pens & SK & ABM & $\mathrm{C}$ \\
\hline 6 & Farm mean number of pigs per drinker in observed pens & PD & $\mathrm{nABM}$ & $\mathrm{C}$ \\
\hline 7 & Farm \% of pens with at least 1 nipple per 12 pigs or 1 water bowl per 15 pigs & PDC & nABM & $\mathrm{C}$ \\
\hline 8 & Farm mean $\%$ of active pigs manipulating enrichment in observed pens & EMB & ABM & $\mathrm{C}$ \\
\hline 9 & Farm mean $\%$ of active pigs in reach of enrichment material in observed pens & PAE & ABM & $\mathrm{C}$ \\
\hline 10 & Farm \% of observed pens with optimal or suboptimal enrichment & EC & $\mathrm{nABM}$ & $\mathrm{C}$ \\
\hline 11 & Farm average number of pigs per farm in 2018 & AVP & nABM & $\mathrm{C}$ \\
\hline 12 & Farm average number of pigs per Annual Work Unit in 2018 & AWU & nABM & $\mathrm{C}$ \\
\hline 13 & Farm maximum pig live weight before slaughter in 2018 & LWS & nABM & $\mathrm{C}$ \\
\hline 14 & Farm Average Daily Growth in 2018 & ADG & ABM & $\mathrm{C}$ \\
\hline 15 & Farm average Feed Conversion Rate in 2018 & FCR & ABM & $\mathrm{C}$ \\
\hline 16 & Farm veterinary and medication per pig sold in 2018 (EUR/pig) & MCP & nABM & $\mathrm{C}$ \\
\hline 17 & Average mortality in 2018 (not including culled pigs) & M & ABM & $\mathrm{C}$ \\
\hline 18 & Farm $\%$ of observed pens with at least one tail lesion in one pig & $\mathrm{T}$ & $\mathrm{ABM}$ & $\mathrm{C}$ \\
\hline 19 & Farm $\%$ of observed pens with at least one ear lesion in one pig & $\mathrm{E}$ & $\mathrm{ABM}$ & $\mathrm{C}$ \\
\hline 20 & Farm $\%$ of observed pens with at least one skin lesion in the body of one pig & B & ABM & $\mathrm{C}$ \\
\hline 21 & Farm presence of tail docked pigs: $0=$ no tail docked; $1=$ some tail docked $; 2=$ all tail docked & TD & nABM & $\mathrm{O}$ \\
\hline 22 & $\begin{array}{l}\text { Farm presence of pigs with short tail: } 0=\text { no pigs with short tail; } 1=\leq 10 \% \text { pigs with short tail; } \\
\qquad 2=>10 \% \text { pigs with short tail }\end{array}$ & SHT & $\mathrm{ABM}$ & $\mathrm{O}$ \\
\hline 23 & $\begin{array}{l}\text { Farm presence of pigs with tail stump: } 0=\text { no pigs with tail stump; } 1=\leq 10 \% \text { pig with tail stump; } \\
\qquad 2=>10 \% \text { pigs with tail stump }\end{array}$ & STT & $\mathrm{ABM}$ & $\mathrm{O}$ \\
\hline 24 & Farm presence of slatted floor: $0=$ no slatted floor; $1=$ partially slatted floor; $2=$ total slatted floor & SF & $\mathrm{nABM}$ & $\mathrm{O}$ \\
\hline 25 & $\begin{array}{l}\text { Farm presence of bedding in lying area: } 0=\text { no bedding, } 1=\text { enough bedding in laying area; } \\
\qquad 2=\text { all pen floor bedded }\end{array}$ & $\mathrm{BP}$ & $\mathrm{nABM}$ & $\mathrm{O}$ \\
\hline 26 & Farm presence of roughage: $0=$ no roughage; $1=$ pellet $; 2=$ straw $; 3=$ hay $/$ silage & $\mathrm{RP}$ & nABM & $\mathrm{O}$ \\
\hline 27 & Farm laying area dirtiness score: $1=$ clean; $2=$ medium; $3=$ dirty & DL & nABM & $\mathrm{O}$ \\
\hline 28 & Farm presence of liquid feeding system: $1=$ dry; $2=$ wet or mixed dry $/$ liquid; $3=$ liquid & LFS & nABM & $\mathrm{O}$ \\
\hline 29 & Organic farm: $0=$ no, $1=$ yes & OR & nABM & $\mathrm{D}$ \\
\hline 30 & Outdoor access: $0=$ no, $1=$ yes & $\mathrm{OA}$ & nABM & $\mathrm{D}$ \\
\hline 31 & Feed restriction: $0=$ no, $1=$ yes & FR & nABM & $\mathrm{D}$ \\
\hline
\end{tabular}

${ }^{1}$ Animal Based Measure (ABM) or non-Animal Based Measure (nABM) ${ }^{2}$ continuous (C) or ordinal (O) or dichotomous (D) variables.

Twenty-three variables were taken into account for the observed pig groups (Table 1): 14 non animal based measures were considered as relevant for pig housing conditions, productivity and management (i.e., four continuous, seven ordinal and three dichotomous variables) together with nine animal-based measures (i.e., four continuous and five ordinal variables), as relevant for the presence or prevalence of pig lesions and for the pig behaviour towards manipulable materials.

Animal welfare measures on 709 pig groups were aggregated as mean values per farm for continuous variables or median values per farm for most ordinal variables, except for variables EP, T, E and B; for each farm the mean value or the median value of pig group observations were considered for each variable. Enrichment presence (EP), tail lesions (T), ear lesions (E) and body lesions (B), as described in Table 1, were transformed from ordinal variables (i.e., score $0,1,2$ ) into continuous variables, as farm percentage of observed pens with optimal or suboptimal enrichment (EC) or with at least one pig with a mild or severe tail (T), ear (E), and body (B) lesion, respectively (Table 2).

The dataset of 51 cases of fattening units was obtained, including eight additional continuous variables (Table 2): six of them related to farm management and productivity, one related to mortality rate (i.e., not including culled, sick or unproductive pigs) in the calendar year before farm observation (i.e., 2018) and one related to the farm percentage of pig groups with at least 1 nipple drinker per 12 pigs or 1 water bowl per 15 pigs.

Management and productivity data were collected in an interview with the farm manager or the person responsible for pig care using the SusPigSys protocol [34]. Data on housing conditions in the pig houses were directly recorded.

As a pig stockperson may be full or part-time employed, the average number of pigs present on a farm (i.e., AVP) was related to the Annual Work Unit (AWU), as defined by 
Eurostat [36] for a stockperson occupied in pig farming on a full-time basis; this variable is the average number of pigs present on a farm in 2018 per AWU (PWU).

Statistical analysis of all data was performed with SPSS Statistics 27, except for Principal Component Analysis (PCA), which was performed using the PLS Toolbox software (v. 8.8.1). The dataset of 51 European pig farms was explored by means of PCA, in order to obtain an overview of the overall data structure, both in terms of correlations between the considered variables and of samples (farms) clustering. The loading plots were used to investigate the relationships between variables. A PCA model was calculated on the whole dataset of pig farms using autoscaling as the variable pre-processing method. Autoscaling consists of transforming each variable by subtracting its average value and then dividing it by its standard deviation. This transformation allows the data to be translated at the origin of the reference system, since each variable will have an average value equal to zero, and also makes the variability of each variable equally important in the construction of the PCA model, since each variable will have standard deviation equal to one [37]. Variables that are close to each other in the loading plot have similar properties and variables that are far apart and are different from each other [38]. The score plots were used both to highlight similarities and differences between the pig farms, and for direct interpretation of the farm cases in relation to variables in the loading plots.

Non-parametric analysis (Kruskal-Wallis test) was applied to the single variables, for not normally distributed data, to further explore the differences between clusters identified by the PCA. Furthermore, pairwise comparisons were performed using the Mann-Whitney U-test, when a significant effect of the farm group was revealed.

\section{Results}

The PCA resulted in four Principal Components (PCs), selected on the basis of the scree plot, explaining $58.43 \%$ of total data variance. The variance not captured by the model can be ascribed to statistical noise due to a number of possible factors such as the biological nature of most data and the limited sensitivity of some variables.

The loading plot of the first two principal components (Figure 1a) shows that PC1, accounting for the largest data variability, is mainly influenced by the variables lying at the left and right extreme parts of the plot.

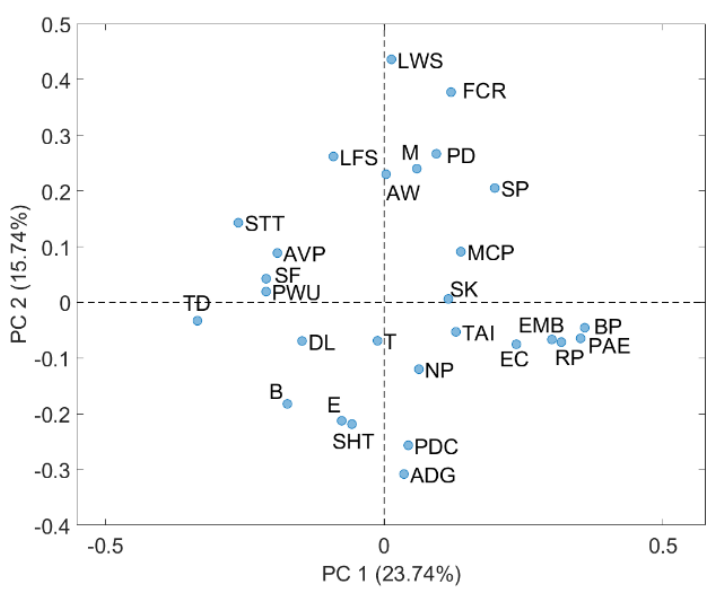

(a)

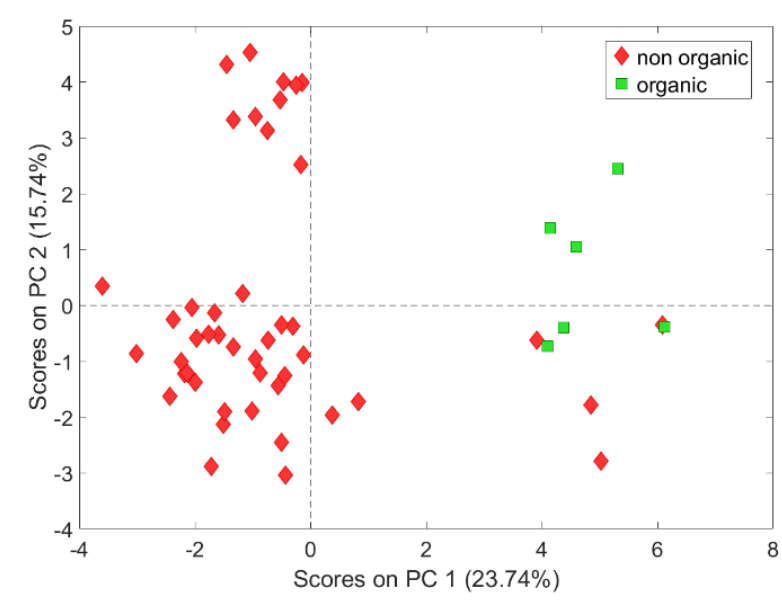

(b)

Figure 1. (a) Loading plot of PC1 and PC2; (b) Score plot of PC1 and PC2, representing organic and non-organic farms.

In particular, on the right side, the following variables are positively correlated with each other and with $\mathrm{PC} 1$ : bedding presence (BP), roughage presence (RP), pig access to enrichment (PAE), enrichment material behaviour (EMB) and percentage of pens with optimal or suboptimal enrichment (EC). These variables are negatively correlated with 
those on the left side of the plot: tail docked pigs (TD), tail stump pigs (STT) and slatted floors (SF).

The score plot of the first two PCs clearly shows three groups of farms located on the right side, on the left topside and on the left downside, respectively (Figure 1b). The group on the right is composed by the only six organic farms in the dataset and by four non organic farms, characterized by some welfare standards similar to the organic ones, according to Regulation (EC) 889/2008 (i.e., bedding in the resting areas, no or limited slatted floor, no or limited tail docking and high space allowance), and above the minimum requirements of Directive 120/2008/EC; this latter group includes two Polish, one German and one Finnish farm (Figure 2).

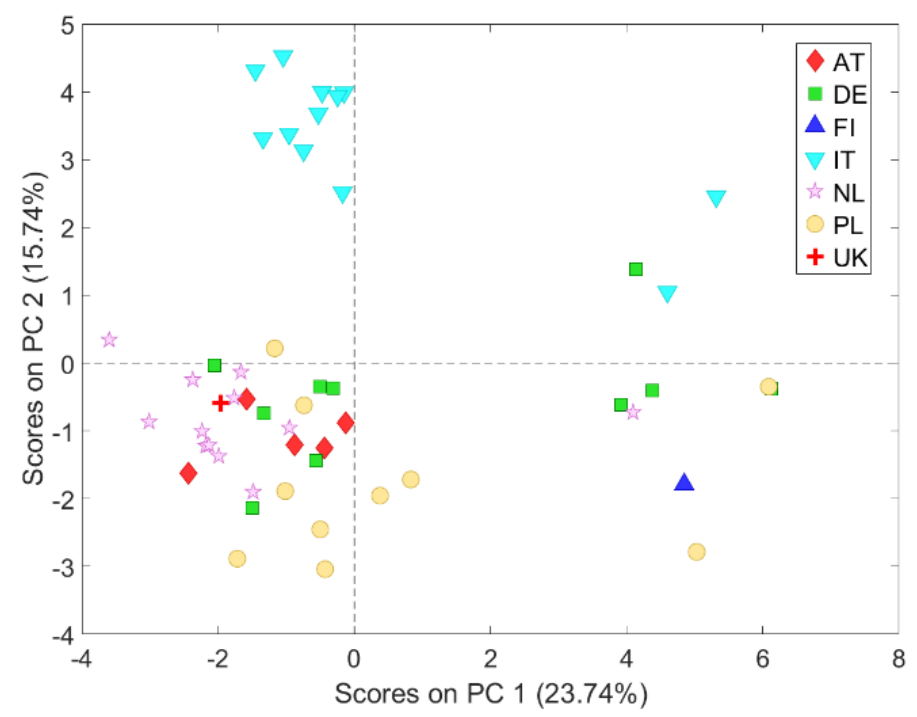

Figure 2. Score plot of PC1 and PC2; farm countries in evidence.

The group on the left topside consists of 10 out of the 12 Italian heavy pig farms (Figure 2), whose positions in the score plot, compared to the corresponding loading plot of Figure 1, reveals that they are characterized by higher live weight at slaughter (LWS), feed conversion ratio (FCR), mortality rate (M), number of pigs per drinker (PD) and the presence of liquid feeding system (LFS). The other two Italian heavy pig farms are organic and represented correctly on the right side of the score plot.

Based on the results of the PCA model, which highlighted the presence of three separate farm clusters (Figure 2), the contribution of each single variable to this grouping was explored by checking for statistically significant differences between clusters:

1. Housing system with bedded solid floored resting area (BED);

2. Housing system with no bedded solid floored resting area for lean pigs (NBL);

3. Housing system with no bedded solid floored resting area for heavy pigs (NBH).

Pen size and number of pigs in the observed pens were not founded statistically different in the three farm groups (Table 3), whereas higher farm average pig live weight (AW) was observed $\left(p=2 \times 10^{-4}\right)$ in NBH farms than in NBL and BED farms, as expected. Lower space allowance per pig (SP) was found $\left(p=6 \times 10^{-6}\right)$ in the NBL farm group, compared to NBH and BED groups. However, lower space allowance per $100 \mathrm{~kg}$ of live weight (SK), was detected in NBH than in NBL and BED; differences are not, though by a small margin, statistically significant $(p=0.067)$ and show that the SK value decreases as the pig live weight increases, particularly in heavy pigs. 
Table 3. Statistics for 20 continuous variables.

\begin{tabular}{|c|c|c|c|c|c|c|c|c|c|c|c|}
\hline \multicolumn{2}{|c|}{ Variables } & \multicolumn{3}{|c|}{ NBH (10 Farms) } & \multicolumn{3}{|c|}{ NBL (31 Farms) } & \multicolumn{3}{|c|}{ BED (10 Farms) } & \multirow[b]{2}{*}{$p$-Value } \\
\hline N. & Acronym & Q25 & Mdn & Q75 & Q25 & Mdn & Q75 & Q25 & Mdn & Q75 & \\
\hline 1 & TAI & 14.2 & 20.6 & 28.9 & 11.5 & 18.2 & 35.0 & 15.6 & 21.1 & 50.1 & $>0.05$ \\
\hline 2 & NP & 12.2 & 17.3 & 25.5 & 14.2 & 21.1 & 37.0 & 14.3 & 19.3 & 40.0 & $>0.05$ \\
\hline 3 & AW & 93.2 & $110.2^{\mathrm{a}}$ & 117.4 & 62.0 & $70.0^{b}$ & 78.2 & 57.4 & $75.0^{b}$ & 90.8 & $<0.01$ \\
\hline 4 & $\mathrm{SP}$ & 1.11 & $1.15^{\mathrm{a}}$ & 1.25 & 0.79 & $0.88^{b}$ & 1.01 & 1.03 & $1.20^{\mathrm{a}}$ & 1.30 & $<0.01$ \\
\hline 5 & SK & 1.09 & 1.24 & 1.57 & 1.15 & 1.40 & 1.63 & 1.26 & 1.77 & 2.25 & $>0.05$ \\
\hline 6 & PD & 10.7 & $14.5^{\mathrm{a}}$ & 25.5 & 7.2 & $8.5^{b}$ & 11.9 & 7.3 & $9.4^{\mathrm{a}, \mathrm{b}}$ & 17.6 & $<0.01$ \\
\hline 7 & PDC & 0.0 & $20^{a}$ & 80.6 & 33.3 & $93^{b}$ & 100.0 & 25.7 & $97^{a, b}$ & 100.0 & $<0.05$ \\
\hline 8 & EMB & 5.5 & $6.8^{a}$ & 13.6 & 4.8 & $12.1^{\mathrm{a}}$ & 21.4 & 41.3 & $72.3^{b}$ & 87.4 & $<0.01$ \\
\hline 9 & PAE & 5.9 & $9.2^{\mathrm{a}}$ & 11.8 & 10.5 & $16.1^{b}$ & 24.5 & 98.7 & $100^{c}$ & 100.0 & $<0.01$ \\
\hline 10 & $\mathrm{EC}$ & 0.0 & $33^{a}$ & 88.3 & 0.0 & $53^{a}$ & 100.0 & 100.0 & $100^{b}$ & 100.0 & $<0.01$ \\
\hline 11 & AVP & 1571 & $2169^{a}$ & 3226 & 976 & $1450^{a}$ & 2419 & 191 & $263^{b}$ & 100.0 & $<0.01$ \\
\hline 12 & AWU & 967 & $1187^{a}$ & 1355 & 444 & $1065^{a}$ & 2182 & 152 & $460^{b}$ & 915 & $<0.05$ \\
\hline 13 & LWS & 165 & $172^{\mathrm{a}}$ & 175 & 118 & $120^{b}$ & 122 & 115 & $122^{b}$ & 140 & $<0.01$ \\
\hline 14 & ADG & 680 & $708^{a}$ & 753 & 800 & $820^{b}$ & 885 & 741 & $780^{b}$ & 1000 & $<0.01$ \\
\hline 15 & FCR & 3.6 & $3.7^{\mathrm{a}}$ & 3.8 & 2.5 & $2.6^{b}$ & 2.8 & 2.8 & $3.0^{\mathrm{c}}$ & 3.5 & $<0.01$ \\
\hline 16 & MCP & 1.4 & $2.6^{\mathrm{a}}$ & 3.4 & 0.5 & $1.0^{b}$ & 2.8 & 1.1 & $2.0^{b}$ & 4.9 & $<0.05$ \\
\hline 17 & $\mathrm{M}$ & 3.3 & $3.8^{\mathrm{a}}$ & 4.6 & 1.5 & $2.0^{b}$ & 2.9 & 2.0 & $2.7^{b}$ & 4.0 & $<0.01$ \\
\hline 18 & $\mathrm{~T}$ & 0.0 & 3.3 & 6.7 & 0.0 & 6.7 & 20.0 & 0.0 & 6.7 & 21.7 & $>0.05$ \\
\hline 19 & $\mathrm{E}$ & 0.0 & $0.0^{\mathrm{a}}$ & 1.7 & 0.0 & $6.7^{\mathrm{b}}$ & 40.0 & 0.0 & $0.0^{a}$ & 0.7 & $<0.05$ \\
\hline 20 & B & 0.0 & $0.0^{\mathrm{a}}$ & 3.3 & 6.7 & $38.5^{b}$ & 73.3 & 0.0 & $0.0^{\mathrm{a}}$ & 8.3 & $<0.01$ \\
\hline
\end{tabular}

TAI, Total area indoor of observed pens; NP, pigs/pen; AW, average pig live weight/pen; SP, space allowance/pig; SK, space allowance/100 $\mathrm{kg}$ pig LW; PD, pigs/drinker; PDC, \% of pens with at least 1 nipple/12 pigs or 1 water bowl $/ 15$ pigs; EMB, $\%$ of active pigs manipulating enrichment; EMB, \% of active pigs manipulating enrichment; PAE, \% of active pigs in reach of enrichment; EC, \% of pens with optimal or suboptimal enrichment; AVP, average number of pigs/farm; AWU, average number of pigs/AWU; LWS, pig live weight at slaughter; ADG, average daily growth; FCR, feed conversion rate; $\mathrm{MCP}$, medication cost/pig; $\mathrm{M}$, mortality rate; $\mathrm{T}, \%$ of pens with at least one pig tail lesion; $\mathrm{E}, \%$ of pens with at least one ear lesion; B, \% of pens with at least one skin lesion. Median (Mdn), lower quartile (Q25) and upper quartile (Q75) values for assessed measures per housing system (i.e., BED, NBL and NBH). $p=$ result of global Kruskal-Wallis test for housing system effect. ${ }^{a, b}, \mathrm{c}$ Median values with different superscripts within a row differ at $p<0.05$ in a pairwise system comparison with Mann-Whitney U-test.

A higher number of pigs per drinker (PD) and lower percentage of pens with at least 1 nipple/12 pigs or 1 water bowl/15 pigs (PDC) were found ( $p=0.003$ and $p=0.044$, respectively) in the NBH group, compared to the NBL group. Farms belonging to the BED group were characterized by higher prevalence $(p=0.001)$ of optimal or suboptimal enrichment (EC) and higher percentage of observed active pigs manipulating (EMB) and in reach (PAE) of enrichment materials ( $p=6 \times 10^{-7}$ and $p=0.001$, respectively), compared to NBH and NBL farms.

The average number of pigs per farm (AVP) and per Annual Work Unit (PWU) in 2018 , were found much lower $\left(p=9 \times 10^{-5}\right.$ and $p=0.024$, respectively) in the BED farm group, than in the NBH and NBL farm groups. Regarding the productive performances, the average daily growth (ADG) was lower $\left(p=4 \times 10^{-4}\right)$ in NBH farms than in the NBL and BED farm groups, whereas the feed conversion rate (FCR) was statistically different in the three groups $\left(p=4 \times 10^{-7}\right)$ and higher NBH farms than in NBL and BED farms.

Average pig mortality rate and medication cost per pig sold were found statistically different from each other in the three farm groups ( $p=0.003$ and $p=0.046$, respectively). No statistically significant difference between the farm percentages of observed pens with at least one tail lesion $(\mathrm{T})$ in the three farm clusters $(p=0.363)$, although all pigs in the NBH and NBL groups were tail docked and most pigs in the BED group (i.e., except in one farm) were tail undocked. Ear and body lesions were detected more frequently in the NBL group than in the NBH and BED groups ( $p=0.023$ and $p=2 \times 10^{-4}$, respectively).

Pigs with tails shortened by less than $50 \%$ of the total tail length (SHT) were found more common in NBL farms than in NBH farms, where most pig tails were shortened by more than $50 \%$ of the total length (STT) (Table 4). Dirtiness in the laying area was 
statistically different $(p=0.008)$ and higher in farms of the NBL group, compared to those if the BED group, but no statistical difference was found between the NBH and BED farm groups. Liquid feeding system was observed as less common in the BED group, compared to the other two farm groups $(p=0.001)$.

Table 4. Statistics for 8 ordinal variables.

\begin{tabular}{cccccccccccc}
\hline \multicolumn{2}{c}{ Variables } & \multicolumn{3}{c}{ NBH (10 Farms) } & \multicolumn{3}{c}{ NBL (31 Farms) } & \multicolumn{3}{c}{ BED (10 Farms) } \\
\hline N. & Acronym & Q25 & Mdn & Q75 & Q25 & Mdn & Q75 & Q25 & Mdn & Q75 & $p$-Value \\
\hline 1 & TD & 1.8 & $2.0^{\mathrm{a}}$ & 2.0 & 2.0 & $2.0^{\mathrm{a}}$ & 2.0 & 0.0 & $0.0^{\mathrm{b}}$ & 0.0 & $<0.01$ \\
2 & SHT & 0.0 & $0.0^{\mathrm{a}}$ & 1.0 & 0.0 & $2.0^{\mathrm{b}}$ & 2.0 & 0.0 & $0.0^{\mathrm{a}}$ & 0.0 & $<0.01$ \\
3 & STT & 2.0 & $2.0^{\mathrm{a}}$ & 2.0 & 0.0 & $2.0^{\mathrm{a}}$ & 2.0 & 0.0 & $0.0^{\mathrm{b}}$ & 0.0 & $<0.01$ \\
4 & SF & 1.0 & $1.5^{\mathrm{a}}$ & 2.0 & 1.0 & $1.0^{\mathrm{a}}$ & 2.0 & 0.0 & $0.5^{\mathrm{b}}$ & 1.0 & $<0.01$ \\
5 & BP & 0.0 & $0.0^{\mathrm{a}}$ & 0.0 & 0.0 & $0.0^{\mathrm{a}}$ & 0.0 & 2.0 & $3.0^{\mathrm{b}}$ & 3.0 & $<0.01$ \\
6 & RP & 0.0 & $0.0^{\mathrm{a}}$ & 0.0 & 0.0 & $0.0^{\mathrm{a}}$ & 0.0 & 2.0 & $2.0^{\mathrm{b}}$ & 2.3 & $<0.01$ \\
7 & DL & 0.0 & $0.0^{\mathrm{a}, \mathrm{b}}$ & 0.3 & 0.0 & $1.0^{\mathrm{a}}$ & 1.0 & 0.0 & $0.0^{\mathrm{b}}$ & 0.0 & $<0.01$ \\
8 & LFS & 2.0 & $2.0^{\mathrm{a}}$ & 2.0 & 0.0 & $0.0^{\mathrm{a}}$ & 2.0 & 0.0 & $0.0^{\mathrm{b}}$ & 1.3 & $<0.01$ \\
\hline
\end{tabular}

TD, tail docked pigs; SHT, pigs with short tails; STT, pigs with tail stump; SF, slatted floor; BP, presence of bedding in the laying area; RP, presence of roughage; DL, dirtiness score in the laying area; LFS, liquid feeding system. Median (Mdn), lower quartile (Q25) and upper quartile (Q75) values for assessed measures per housing system (i.e., BED, NBL and NBH). $p=$ result of global Kruskal-Wallis test for housing system effect. ${ }^{a, b}$ Median values with different superscripts within a row differ at $p<0.05$ in a pairwise system comparison with Mann-Whitney U-test.

The organic farms in the dataset are all included in the BED group. Most farms allowing pigs to access outdoors are also in the BED farm group; only one of them belongs to the NBL group. Feed restriction is used in $90 \%$ of the NBH farms and in only $29 \%$ and $30 \%$ of the NBL and BED farms, respectively (Table 5).

Table 5. Descriptive statistics for 3 dichotomous variables; percentage of farms in the group.

\begin{tabular}{cccccc}
\hline $\mathbf{N}$ & $\begin{array}{c}\text { Variable } \\
\text { Description }\end{array}$ & Acronym & NBH \% & NBL \% & BED \% \\
\hline 1 & Organic farm & OR & 0 & 0 & 60 \\
2 & Outdoor access & OA & 0 & 3 & 70 \\
3 & Feed restriction & FR & 90 & 29 & 30 \\
\hline
\end{tabular}

\section{Discussion}

Lower farm size (AVP) and number of pigs per Annual Work Unit (PWU) were found in farms of the BED group, compared to farms of the other two clusters, suggesting that the higher labour needed per pig in these farms could be related to a higher workload for the management of bedding materials, as well as to less economies of scale in place in these smaller sized farms. These farms are featured by no or limited slatted floor, presence of proper manipulable material and roughage, higher space allowance and no or limited tail docking; almost all pigs observed in these farms were found in reach of optimal enrichment materials, according to the EC Recommendation 336/2016 [23], and most active pigs were manipulating them in the observed pens. Lower prevalence of ear and body lesions was found in these farms, compared to lean pig farms without bedded solid floored resting area (NBL), but not compared to heavy pig farms without bedded solid floored resting area $(\mathrm{NBH})$, where the higher age of the observed pigs and the higher pig space allowance could have mitigated the occurrence of these lesions.

The group of heavy pig farms without bedded solid floored resting area (NBH) is characterized by the presence of fully or partially slatted floor, liquid feeding, and limited availability of drinkers, which is considered as a risk factor for pig welfare, particularly in summertime when the water nutritional need tends to increase [16], as well as the competition of pigs to access water. 
Higher space allowance detected in the NBH and BED groups, compared to the HBL group, can be related to the higher live weight in NBH farms and the higher space allowance needed to house pigs with bedded solid floored resting area. It is worth noting that the pig space allowance of $1.15 \mathrm{~m}^{2} /$ pig in the heavy pig farms of the NBH group exceeds the minimum legal requirement of $1 \mathrm{~m}^{2} / \mathrm{pig}$ for pigs over the live weight of $110 \mathrm{~kg}$, although it is lower than the value on $1.48 \mathrm{~m}^{2} / \mathrm{pig}$, calculated through the allometric formula for pigs of $172 \mathrm{~kg}$ of live weight with $\mathrm{k}=0.047$, as recommended by EFSA [10]. However, more space allowance for heavy pigs could be further used in the next few years by heavy pig farmers in an attempt to house pigs with intact tails, in compliance with EU legislation.

The lower presence of roughage and of bedding material in the laying area in farms of the NBH e and NBL groups, compared to the BED group, can also be related to the higher prevalence of slatted floor, which is likely to limit the use of a large quantity of organic materials (e.g., straw, wood shavings) because of the inability of the most common slurry systems (e.g., vacuum system) in place in European fattening pig farms to handle and evacuate these organic materials, together with the liquid manure under the slats, as confirmed in previous studies [2]. The relatively low dirtiness score in the laying area of NBH heavy pig farms can be related to the presence slatted floor in these farms.

Lean pig farms without bedded solid floored resting area (NBL) were featured by lower pig space allowance, mortality, medication cost, number of pigs per drinker and feed conversion rate and by higher average daily growth and prevalence of ear and body lesions, compared to NBH heavy pig farms.

No statistical difference was found between the farm percentages of observed pens with at least one tail lesion in one pig in the three farm groups, although tail docking was performed in both NBH and NBL farms on almost all pigs and not performed on most pigs in BED farms; this outcome suggests that the prevalence of tail biting in undocked tail pigs can be similar to docked tail pigs housed in intensive systems, if undocked tail pigs are housed with bedded solid floored resting area, plenty of manipulable material that most pigs are able to access, and larger space allowance above the minimum EU legal requirements.

Low prevalence of tail lesions in the NBH group can be related to the majority of pigs with tails shortened by more than 50\% (STT), which is likely to expose the pigs to less severe tail lesions but also to more painful tail docking and more frequent formation of neuromas afterwards [26]. Low prevalence of tail lesions in NBH farms can be explained by the higher liveweight (AW) and age of the observed pigs, confirming the outcomes of a previous study showing more severe tail lesions in younger pigs, compared to older ones [39].

The observation of more prevalent ear and skin lesions in pigs of the NBL farm group could be ascribed to the lower space allowance at the start of the growing phase when more frequent fights may occur in recently grouped pigs to establish a hierarchy [40]. Higher pig mortality rates and medication costs per pig in NBH heavy pig farms can be explained by the longer duration of the fattening period and the higher age at slaughter of at least nine months in heavy pigs for Parma Ham PDO. Mortality rate and medication cost per pig could also be biased by different culling rates across farms, due to a different degree of implementing this practice to reduce animal suffering and the spread of diseases on farms.

\section{Conclusions}

In conclusion, the set of animal and non-animal-based measures used in this study, was found suitable and useful to assess, analyse, and compare most of the housing risk factors for pig welfare on farms. Greater validity of the statistical model used in this study could result from a greater availability in the future of pig farm cases in the SusPigSys database.

Additional non-animal-based measures of pig welfare could also be considered to assess the microclimate pig comfort and the presence on noxious gases and dust as risk factors for tail biting and for overall welfare assessment. Animal-based indicators were 
used to monitor pig welfare directly and to investigate the effect that the resources and management have on the animals. Both animal and non-animal-based measures provided different types of information, which are needed for routine official controls and are suitable for use in farm assurance schemes.

Housing risk factors for pig welfare, such as the lack of space allowance, bedding, and environmental enrichment, as well as the presence of fully slatted floor and the availability of drinkers to ensure pigs have permanent access to drinkable water are likely to become more challenging for pig farmers to keep pigs intensively with long undocked tails, once the ban of routine tail docking is finally applied across all EU Member States.

Author Contributions: Conceptualization, P.F.; methodology, P.F.; software, P.F.; validation, A.U.; formal analysis, P.F.; investigation, P.F.; resources, P.F.; data curation, P.F.; writing-original draft preparation, P.F.; writing-review and editing, P.F., A.U. and M.B.; visualization, P.F.; supervision, M.B.; project administration, P.F. and M.B.; funding acquisition, P.F. All authors have read and agreed to the published version of the manuscript.

Funding: This research was made possible by funding from SusAn, an ERA-Net co-funded under European Union's Horizon 2020 research and innovation programme (www.era-susan.eu accessed on 9 October 2021), under Grant Agreement $n^{\circ}$ 696231. The SusPigSys project was coordinated by Sabine Dippel.

Institutional Review Board Statement: Ethical review and approval were waived for this study, due to the observational nature of this study not entailing any harm to the observed animals (i.e., pigs kept under commercial conditions).

Informed Consent Statement: Informed consent was obtained from all subjects involved in the study.

Data Availability Statement: The data presented in this study are openly available in FigShare at https:/ / doi.org/10.6084/m9.figshare.16726567 (accessed on 9 October 2021).

Acknowledgments: The authors are grateful to Rachel Chapman (UK), Claudio Montanari (IT), Monika Gebska and Agata Malak-Rawlikowska (PL), Juliane Helmerichs (DE), Antonia Ruckli (AT), Kirsi Swan (FI), and Herman Vermeer (NL).

Conflicts of Interest: The authors declare no conflict of interest.

\section{References}

1. European Commission. Council Directive 91/630/EEC of 19 November 1991 laying down minimum standards for the protection of pigs. Off. J. Eur. Communities 1991, 340, 33-38.

2. Pedersen, L.J. Overview of Commercial Pig Production Systems and Their Main Welfare Challenges. In Advances in Pig Welfare; Woodhead Publishing Series in Food Science, Technology and Nutrition; Aarhus University: Tjele, Denmark, 2018 ; pp. 3-25. [CrossRef]

3. Averós, X.; Aparicio, M.A.; Ferrari, P.; Guy, J.H.; Hubbard, C.; Schmid, O.; Ilieski, V.; Spoolder, H.A.M. The effect of steps to promote higher levels of farm animal welfare across the EU. Societal versus animal scientists' perceptions of animal welfare. Animals 2013, 3, 786-807. [CrossRef] [PubMed]

4. Welfare Quality ${ }^{\circledR}$. Welfare Quality ${ }^{\circledR}$ Assessment Protocol for Pigs (Sows and Piglets, Growing and Finishing Pigs); Welfare Quality®Consortium: Lelystad, The Netherlands, 2009.

5. EFSA. Scientific Opinion of the Panel on Animal Health and Welfare on the use of animal-based measures to assess welfare in pigs. EFSA J. 2012, 10, 2512. [CrossRef]

6. Temple, D.; Courboulay, V.; Manteca, X.; Velarde, A.; Dalmau, A. The welfare of growing pigs in five different production systems: Assessment of feeding and housing. Animal 2012, 6, 656-667. [CrossRef]

7. Temple, D.; Courboulay, V.; Velarde, A.; Dalmau, A.; Manteca, X. The welfare of growing pigs in five different production systems in France and Spain: Assessment of health. Anim. Welf. 2012, 21, 257-271. [CrossRef]

8. Consortium of Parma Ham. Economic Figures. Available online: https://www.prosciuttodiparma.com/en/parma-hamconsortium/ (accessed on 5 August 2021).

9. Spoolder, H.A.M.; Edwards, S.A.; Corning, S. Legislative methods for specifying stocking density and consequences for the welfare of finishing pigs. Livest. Prod. Sci. 2000, 64, 167-173. [CrossRef]

10. EFSA. Opinion of the Scientific Panel on Animal Health and Welfare on a request from the Commission related to welfare of weaners and rearing pigs: Effects of different space allowances and floor types. EFSA J. 2005, 268, 1-19. [CrossRef]

11. Vermeer, H.M.; De Greef, K.H.; Houwers, H.W.J. Space allowance and pen size affect welfare indicators and performance of growing pigs under Comfort Class conditions. Livest. Sci. 2014, 159, 79-86. [CrossRef] 
12. Spoolder, H.A.M.; Edwards, S.A.; Corning, S. Effects of group size and feeder space allowance on welfare in finishing pigs. Anim. Sci. 1999, 69, 481-489. [CrossRef]

13. Turner, S.P.; Allcroft, D.J.; Edwards, S.A. Housing pigs in large social groups: A review of implications for performance and other economic traits. Livest. Prod. Sci. 2003, 82, 39-51. [CrossRef]

14. D'Eath, R.B.; Arnott, G.; Turner, S.P.; Jensen, T.; Lahrmann, H.P.; Busch, M.E.; Niemi, J.K.; Lawrence, A.B.; Sandøe, P. Injurious tail biting in pigs: How can it be controlled in existing systems without tail docking? Animal 2014, 8, 1479-1497. [CrossRef]

15. Meyer-Hamme, S.E.K.; Lambertz, C.; Gauly, M. Does group size have an impact on welfare indicators in fattening pigs? Animal 2016, 10, 142-149. [CrossRef]

16. EFSA. Scientific Opinion of the Panel on Animal Health and Welfare on a request from the Commission on Animal health and welfare in fattening pigs in relation to housing and husbandry. EFSA J. 2007, 564, 1-14. [CrossRef]

17. Battini, M.; Tremolada, C.; Ferrari, L.; Borciani, M.; Gastaldo, A.; Barbieri, S. Straw bedding housing for growing pigs: Effect of two different management systems on hygiene and welfare. Large Anim. Rev. 2016, 22, 225-229.

18. Jensen, T.; Kold Nielsen, C.; Vinther, J.; D'Eath, R.B. The effect of space allowance for finishing pigs on productivity and pen hygiene. Livest. Sci. 2012, 149, 33-40. [CrossRef]

19. Vitali, M.; Nannoni, E.; Sardi, L.; Martelli, G. Knowledge and Perspectives on the Welfare of Italian Heavy Pigs on Farms. Animals 2021, 11, 1690. [CrossRef] [PubMed]

20. European Commission. Council Directive 2008/120/EC of 18 December 2008 laying down minimum standards for the protection of pigs (Codified version). Off. J. Eur. Union 2009, 47, 5-13.

21. Rossi, P.; Gastaldo, A. Acqua di bevanda. In Ricoveri Attrezzature e Impianti per l'Allevamento dei Suini; Edizioni L'Informatore Agrario: Verona, Italy, 2004; Volume 6, pp. 139-154.

22. EFSA AHAW Panel. Scientific Opinion concerning a multifactorial approach on the use of animal and non-animal-based measures to assess the welfare of pigs. EFSA J. 2014, 12, 3702. [CrossRef]

23. European Union. Commission Recommendation (EU) 2016/336 on the application of Council Directive 2008/120/EC laying down minimum standards for the protection of pigs as regards measures to reduce the need for tail-docking. Off. J. Eur. Union 2016, 62, 20-22.

24. Wallgren, T.; Gunnarsson, S. Effect of straw provision in racks on tail lesions, straw availability, and pen hygiene in finishing pigs. Animals 2021, 11, 379. [CrossRef]

25. Scollo, A.; Gottardo, F.; Contiero, B.; Edwards, S.A. A cross-sectional study for predicting tail biting risk in pig farms using classification and regression tree analysis. Prev. Vet. Med. 2017, 146, 114-120. [CrossRef]

26. EFSA. Scientific Opinion of the Panel on Animal Health and Welfare on a request from Commission on the risks associated with tail biting in pigs and possible means to reduce the need for tail-docking considering the different housing and husbandry systems. EFSA J. 2007, 611, 1-13. [CrossRef]

27. Kongsted, H.; Sørensen, J.T. Lesions found at routine meat inspection on finishing pigs are associated with production system. Vet. J. 2017, 223, 21-26. [CrossRef] [PubMed]

28. Alban, L.; Petersen, J.V.; Busch, M.E. A comparison between lesions found during meat inspection of finishing pigs raised under organic/free-range conditions and conventional, indoor conditions. Porc. Health Manag. 2015, 1, 4. [CrossRef] [PubMed]

29. Sørensen, J.T. A comment on the paper 'A comparison between lesions found during meat inspection of finishing pigs raised under organic/free-range conditions and conventional indoor conditions' by Alban et al. 2015. Porc. Health Manag. 2016, 2, 14. [CrossRef] [PubMed]

30. De Briyne, N.; Berg, C.; Blaha, T.; Palzer, A.; Temple, D. Phasing out pig tail docking in the EU—Present state, challenges and possibilities. Porc. Health Manag. 2018, 4, 27. [CrossRef]

31. Dippel, S.; Leeb, C.; Bochicchio, D.; Bonde, M.; Dietze, K.; Gunnarsson, S.; Lindgren, K.; Sundrum, A.; Wiberg, S.; Winckler, C.; et al. Health and welfare of organic pigs in Europe assessed with animal-based parameters. Org. Agric. 2014, 4, 149-161. [CrossRef]

32. Saxmose Nielsen, S.; Alvarez, J.; Bicout, D.J.; Calistri, P.; Depner, K.; Drewe, J.A.; Garin-Bastuji, B.; Gonzales Rojas, J.L.; Gortázar Schmidt, C.; Michel, V.; et al. Welfare of pigs during killing for purposes other than slaughter. EFSA J. 2020, 18 , e06195. [CrossRef]

33. Costa, F.A.D.; Gibson, T.J.; Oliveira, S.E.O.; Gregory, N.G.; Coldebella, A.; Faucitano, L.; Costa, O.A.D. On-farm pig dispatch methods and stockpeople attitudes on their use. Livest. Sci. 2019, 221, 1-5. [CrossRef]

34. The SusPigSys Team. Condensed Protocol from Era-Net SusAn Project "Sustainable Pig Production Systems" (SusPigSys). A Starting Point for Connecting Data Bases for Integrated Sustainability Assessment. Deliverable 5.1 2020v. Available online: https:/ / www.researchgate.net/publication/348466379_Condensed_protocol_from_Era-Net_SusAn_project_Sustainable_ pig_production_systems_SusPigSys (accessed on 9 October 2021).

35. Pandolfi, F.; Stoddart, K.; Wainwright, N.; Kyriazakis, I.; Edwards, S. The 'Real Welfare' scheme: Benchmarking welfare outcomes for commercially farmed pigs. Animal 2017, 11, 1816-1824. [CrossRef]

36. Eurostat Statistics Explained. Available online: https:/ / ec.europa.eu/eurostat/statistics-explained/index.php?title=Glossary: Annual_work_unit_(AWU) (accessed on 1 November 2021).

37. Wise, B.M.; Gallagher, N.B. The process chemometrics approach to process monitoring and fault detection. J. Process Control 1996, 6, 329-348. [CrossRef] 
38. Næs, T.; Brockhoff, P.B.; Tomic, O. Principal Component Analysis. In Statistics for Sensory and Consumer Science; John Wiley and Sons: Hoboken, NJ, USA, 2010; Volume 14, pp. 209-225.

39. Munsterhjelm, C.; Heinonen, M.; Valros, A. Effects of clinical lameness and tail biting lesions on voluntary feed intake in growing pigs. Livest. Sci. 2015, 181, 210-219. [CrossRef]

40. Turner, S.P.; Farnworth, M.J.; White, I.M.S.; Brotherstone, S.; Mendl, M.; Knap, P.; Penny, P.; Lawrence, A.B. The accumulation of skin lesions and their use as a predictor of individual aggressiveness in pigs. Appl. Anim. Behav. Sci. 2006, 96, 245-259. [CrossRef] 